\title{
Simulations of Liquid Ammonia Based on the Combined Quantum Mechanical/Molecular Mechanical (QM/MM) Approach
}

\author{
Anan Tongraar,*,† Teerakiat Kerdcharoen, ${ }^{\ddagger}$ and Supot Hannongbua ${ }^{\S}$ \\ School of Chemistry, Institute of Science, Suranaree University of Technology, Nakhon Ratchasima 30000, \\ Thailand, Physics Department and Center of Nanoscience and Nanotechnology, Faculty of Science, \\ Mahidol University, Bangkok 10400, Thailand, Department of Chemistry, Faculty of Science, \\ Chulalongkorn University, Bangkok 10330, Thailand
}

Received: December 16, 2005; In Final Form: February 12, 2006

\begin{abstract}
Two combined quantum mechanics/molecular mechanics (QM/MM) molecular dynamics simulations, namely, HF/MM and B3LYP/MM, have been performed to investigate the local structure and dynamics of liquid ammonia. The most interesting region, a sphere containing a central reference molecule and all its nearest surrounding molecules (first coordination shell), was treated by the Hartree-Fock (HF) and hybrid density functional B3LYP methods, whereas the rest of the system was described by the classical pair potentials. On the basis of both HF and B3LYP methods, it is observed that the hydrogen bonding in this peculiar liquid is weak. The structure and dynamics of this liquid are suggested to be determined by the steric packing effects, rather than by the directional hydrogen bonding interactions. Compared to previous empirical as well as Car-Parrinello (CP) molecular dynamics studies, our QM/MM simulations provide detailed information that is in better agreement with experimental data.
\end{abstract}

\section{Introduction}

Hydrogen-bonded liquids, in particular water, are of fundamental scientific interest because of their importance in determining the structure and reactivity of biological molecules. ${ }^{1-3}$ In addition to liquid water, ammonia $\left(\mathrm{NH}_{3}\right)$ is another interesting liquid, which has long been the subject of a number of experimental and theoretical studies..$^{4-17}$ The structure of liquid $\mathrm{NH}_{3}$ has been derived from X-ray ${ }^{4}$ and neutron diffraction measurements. ${ }^{5}$ However, only the total structure factor and the total radial distribution function (RDF) were extracted from these measurements. The nature of the hydrogen bonds in the liquid was inferred from a comparison with known solid structure. Therefore, these experiments cannot be considered as being conclusive. Later, a complete set of neutron diffraction experiments with isotropic H/D substitution (NDIS) were done for the liquids $\mathrm{NH}_{3}, \mathrm{ND}_{3}$ and an isomolar $\mathrm{NH}_{3} / \mathrm{ND}_{3}$ mixture at 213 and $273 \mathrm{~K} .{ }^{7}$ This allowed for an investigation of the orientational correlations as well as the degree of hydrogen bond in the liquid. According to the NDIS experiments, it has been demonstrated that no hydrogen-bonded network exists in the liquid $\mathrm{NH}_{3}$. Some degrees of hydrogen bonding in the liquid could be inferred from the temperature dependence of the $\mathrm{N}-\mathrm{H}$ and $\mathrm{H}-\mathrm{H}$ RDFs. Nevertheless, these hydrogen-bond interactions are much weaker than that in liquid water.

In conjunction with these experiments, computer simulations have provided structural and dynamical details of liquid $\mathrm{NH}_{3}$ at molecular level.$^{8-17}$ Most earlier studies relied on simplified potentials ${ }^{8-14}$ or on direct ab initio pair interactions, ${ }^{15}$ which all lead to questionable agreement with experimental observations. Later simulation, ${ }^{15}$ where the pair interactions were

* Corresponding author. E-mail: anan_tongraar@yahoo.com. Fax:006644-224185.

Suranaree University of Technology.

$\doteqdot$ Mahidol University.

$\S$ Chulalongkorn University. directly calculated on the basis of ab initio calculation suggested that the discrepancy between the experimental and theoretical investigation in the first solvation shell is due to many-body effects, particularly the three-body effects. A pioneer attempt to obtain a reliable picture of liquid $\mathrm{NH}_{3}$ was based on the CarParrinello molecular dynamics (CP-MD) method. ${ }^{18,19}$ The CPMD derived results were found to be in better agreement with experimental data than those of the earlier studies using empirical fitted potentials. ${ }^{16,17}$ However, this approach was applied to a relatively small system, i.e., a replicated cubic box made up of only 32 ammonia molecules, and all interactions were evaluated by simple BLYP functional, which leads to a reduction in the accuracy. For example, the simulation by Tuckerman and co-workers, ${ }^{16}$ although providing a first $\mathrm{N}-\mathrm{N}$ peak with maximum and average coordination number in good agreement with the NDIS data, apparently overestimated the hydrogen-bonded structure as well as predicted narrower and more intense $\mathrm{N}-\mathrm{N}$ RDF. Furthermore, a subsequent $\mathrm{CP}-\mathrm{MD}$ study ${ }^{20}$ of liquid $\mathrm{NH}_{3}$ containing ammonium or amide ion has reported somewhat different results for the $\mathrm{N}-\mathrm{N}$ and $\mathrm{N}-\mathrm{H}$ correlations between the bulk ammonia molecules when compared to the earlier study. ${ }^{16}$ Recently, a novel HCTH407+ functional was developed for describing the characteristics of hydrogen bond on the level of dimer and was used in the CPMD simulations of liquid $\mathrm{NH}_{3} \cdot{ }^{17}$ It has been demonstrated that this newly developed HCTH407+ functional reproduces a nonhydrogen-bonded first shell peak that is in good agreement with the corresponding peak observed in experiments. It was pointed out, however, that this functional significantly overestimated the equilibrium $\mathrm{N}-\mathrm{N}$ distance. In addition, for the hydrogenbonded part, this functional yielded a less pronounced $\mathrm{N}-\mathrm{H}$ shoulder peak, in comparison to experimental data.

An alternative approach for the treatment of such weakly interacting system is to employ a so-called combined quantum mechanical/molecular mechanical (QM/MM) molecular dynam- 
ics technique. ${ }^{21-24}$ This simulation technique has been proven to be a very reliable simulation method. It provides more informative insights into the structure and dynamics of various condensed-phase systems, in particular ionic solutions. ${ }^{25-34}$ Very recently, a QM/MM simulation has been performed for pure water ${ }^{35}$ that compares the performance of the second-order Møller-Plesset perturbation theory (MP2), the Hartree-Fock (HF) and the density functional theory (DFT) methods. It has been demonstrated that the HF- and MP2-based simulations provided detailed information on pure water in which are in good agreement with most recent experimental data. The DFT method, even with the B3LYP functional, has predicted improper dynamics due to too rigid hydrogen bonds. In the present work, it is of particular interest to evaluate the validity and accuracy of quantum mechanical methods in describing the structure and dynamics of the weakly bound liquid $\mathrm{NH}_{3}$. Because the high correlated methods, like the MP2, are far too time-consuming, the HF and hybrid density functional B3LYP methods are thus only the possible alternatives of the present study.

\section{Methods}

For the QM/MM approach, ${ }^{25-35}$ the system is divided into two parts, namely QM and MM regions. The total interaction energy of the system is then defined as

$$
E_{\text {total }}=\left\langle\Psi_{\mathrm{QM}}|\hat{H}| \Psi_{\mathrm{QM}}\right\rangle+E_{\mathrm{MM}}+E_{\mathrm{QM}-\mathrm{MM}}
$$

where $\left\langle\Psi_{\mathrm{QM}}|\hat{H}| \Psi_{\mathrm{QM}}\right\rangle$ refers to the interactions within the QM region and $E_{\mathrm{MM}}$ and $E_{\mathrm{QM}-\mathrm{MM}}$ represent the interactions within the $\mathrm{MM}$ and between the QM and MM regions, respectively. The QM region, the most interesting part of which includes a central ammonia molecule and almost all nearest surrounding molecules lying in the first coordination shell, is treated quantum mechanically using the HF and B3LYP methods. The rest of the system is described by the classical $\mathrm{NH}_{3}-\mathrm{NH}_{3}$ pair potentials. ${ }^{11,36}$ In general, the post-HF methods with the extended basis sets are most suitable for the treatment of hydrogen-bonded systems. It turns out, however, to be computationally prohibitive. In practice, some essential parameters such as quantum mechanics level of theory, basis set and size of QM region must be optimized, making a compromise between the quality of the simulation results and the requirement of CPU time. The MP2based method is far beyond the available computational resources, and so only the HF and B3LYP methods were employed for describing all interactions within the defined QM region. All quantum mechanical calculations were carried out using double- $\zeta$ plus polarization (D95*) basis set. ${ }^{37}$ To define the size of the QM region, a classical pair potential simulation was performed. A radius of $5.0 \AA$ from central ammonia corresponding to the first minimum of the $\mathrm{N}-\mathrm{N}$ radial distribution function was chosen (e.g., see inset in Figure 1). This QM size contains $12-16$ ammonia molecules, covering the first shell coordination numbers predicted by almost all experimental and theoretical reports. ${ }^{4-15}$ This QM region was considered large enough, requiring approximately $8-14 \mathrm{~min}$ to compute quantum mechanical forces in each QM/MM MD step (on an Intel Pentium IV $2.8 \mathrm{GHz}$ processor).

During the QM/MM simulations, exchange of ammonia molecules between the QM and $\mathrm{MM}$ regions took place frequently. In regard to this point, the forces acting on each particle in the system were switched according to which region the ammonia molecule was entering or leaving the QM region and is defined as

$$
F_{i}=S_{m}(r) F_{\mathrm{QM}}+\left(1-S_{m}(r)\right) F_{\mathrm{MM}}
$$

where $F_{\mathrm{QM}}$ and $F_{\mathrm{MM}}$ are quantum mechanical and molecular mechanical forces, respectively. $S_{m}(r)$ is a smoothing function ${ }^{38}$ described by

$$
\begin{array}{ll}
S_{m}(r)=1 & \text { for } r \leq r_{1} \\
S_{m}(r)=\frac{\left(r_{0}{ }^{2}-r^{2}\right)^{2}\left(r_{0}{ }^{2}+2 r^{2}-3 r_{1}{ }^{2}\right)}{\left(r_{0}{ }^{2}-r_{1}{ }^{2}\right)^{3}} & \text { for } r_{1}<r \leq r_{0} \\
S_{m}(r)=0 & \text { for } r>r_{0}
\end{array}
$$

where $r_{1}$ and $r_{0}$ are the distances characterizing the start and the end of the QM region, applied within an interval of $0.2 \AA$ (i.e., between the $\mathrm{N}-\mathrm{N}$ distance of $5.0-5.2 \AA$ ) to ensure a continuous change of forces at the transition between $\mathrm{QM}$ and MM regions.

All MD simulations were performed in a canonical ensemble at $273 \mathrm{~K}$ with periodic boundary conditions. The system's temperature was kept constant using the Berendsen algorithm. ${ }^{39}$ A periodic box, with a box length of $21.80 \AA$, containing 256 ammonia molecules, corresponding to the experimental density of pure ammonia $\left(0.6988 \mathrm{~g} \mathrm{~cm}^{-3}\right)$ was used. The reaction-field method $^{40}$ was employed for the treatment of long-range interactions. The Newtonian equations of motions were treated by a general predictor-corrector algorithm. The time step size was set to $0.2 \mathrm{fs}$, which allows for the explicit movement of the hydrogen atoms of ammonia molecules. The system was initially equilibrated by performing a classical pair potential simulation for 200000 time steps. Then, the HF/MM and B3LYP/MM simulations were started independently with the system's reequilibration for 25000 time steps. Then, a further 90000 and 70000 time steps were continued for the HF/MM and B3LYP/MM simulations, respectively, to collect the configurations every 10 th step.

\section{Results and Discussion}

3.1. Structural Data. The local structure of liquid $\mathrm{NH}_{3}$ is characterized through the $\mathrm{N}-\mathrm{N}$ radial distribution functions (RDFs) and their corresponding integration numbers, as shown in Figures 1 and 2. The first atom in the RDFs refers to the atom of the central reference molecule. The latter represents the atom of other ammonia molecules. In Figures 1 and 2 (together with Figures 4 and 5), the available experimentally observed structures of the liquid $\mathrm{NH}_{3}$ as well as the results obtained by classical pair potential simulation (see insertions) are also given for comparison. For the N-N RDFs, both HF/ $\mathrm{MM}$ and B3LYP/MM simulations show nonsymmetrical first peaks at 3.59 and $3.43 \AA$, respectively. Integrations up to their corresponding first $\mathrm{N}-\mathrm{N}$ minima at 5.30 and $5.20 \AA$ yielded average coordination numbers of 13.6 and 13.3, respectively. These values are in good agreement with the experimental data for about 14 ammonia molecules. ${ }^{7}$ In comparison to the double structured first $\mathrm{N}-\mathrm{N}$ peak extracted from X-ray diffraction ${ }^{4}$ (Figure 1), both the HF/MM and B3LYP/MM simulations predict significant difference in the shape and height of the first $\mathrm{N}-\mathrm{N}$ RDFs. The B3LYP/MM's N-N RDF starts to be detected earlier whereas the distance to its maximum of $3.43 \AA$ appears at the same distance when compared to the X-ray results. This differs from the HF/MM's N-N RDF in that although the shape is consistent with the $\mathrm{X}$-ray result at short distance, its maximum is around $0.2 \AA$ longer than that observed from the X-ray study. The difference between the B3LYP/MM's and the HF/MM's 


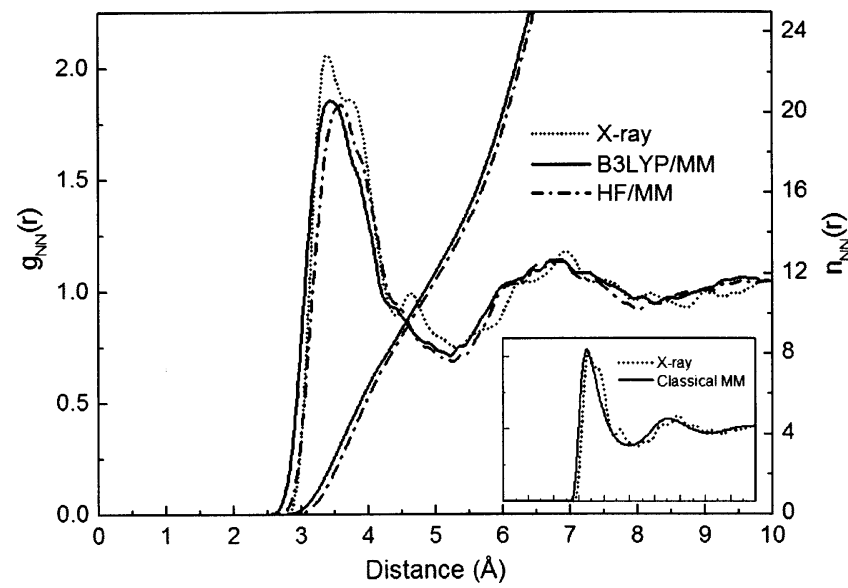

Figure 1. $\mathrm{N}-\mathrm{N}$ radial distribution functions and their corresponding integration numbers, comparing the results obtained from the simulations and the X-ray diffraction experiment.

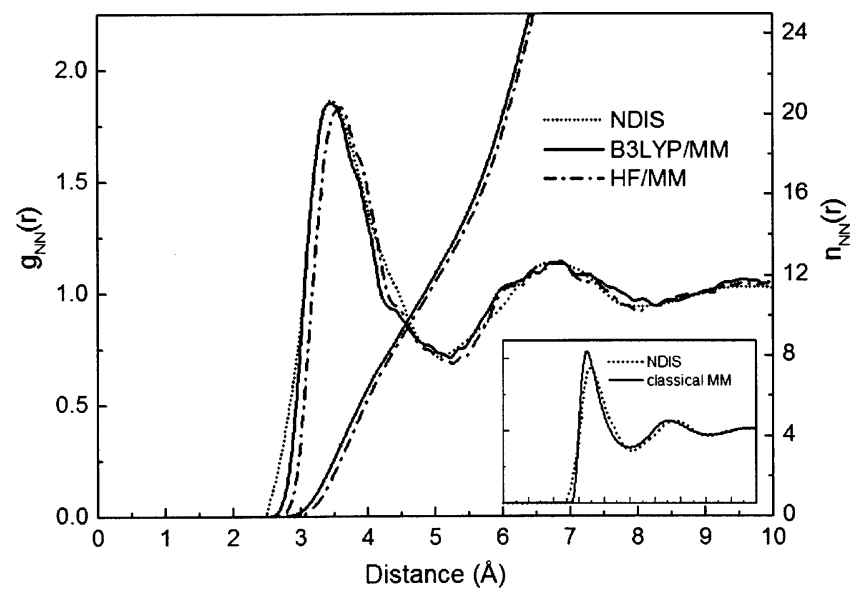

Figure 2. $\mathrm{N}-\mathrm{N}$ radial distribution functions and their corresponding integration numbers, comparing the results obtained from the simulations and the NDIS experiment.

$\mathrm{N}-\mathrm{N}$ RDFs was significant, especially in the first solvation shell where the first appears at a distance about $0.2 \AA$ shorter than the second one. This result leads to the conclusion that inclusion of the electron correlation reflected in less repulsion of ammonia molecules in their close packed structures.

Better agreement is seen when the simulated N-N RDFs are compared to the NDIS data ${ }^{7}$ (Figure 2). It is known that the $\mathrm{X}$-ray data mainly provide the correlations between nitrogen atoms, whereas more knowledge on the three partial radial correlation functions can be obtained from the NDIS experiment. According to the NDIS data, the more intense $\mathrm{N}-\mathrm{N}$ peak observed in the X-ray diffraction experiment arises from the inaccuracy of the X-ray scattering factors calculated for the $\mathrm{NH}_{3}$. It has been shown that the X-ray structures at 3.7 and at $4.6 \AA$ are artifacts of the data analysis. In our study, the B3LYP/MM's $\mathrm{N}-\mathrm{N}$ RDF agrees very well with the NDIS data. The discrepancy at short distance, less than $2.8 \AA$ where the NDIS RDF starts to be observed earlier, is not yet understood. The effect of electron correlation embedded in the HF/MM simulation shifts the $\mathrm{N}-\mathrm{N}$ RDF significantly toward longer distance. Another interesting feature of the simulated N-N RDFs can be recognized from the broad shoulders occurring at the distances between 3.7 and $4.2 \AA$. In the NDIS experiment, ${ }^{7}$ the corresponding $\mathrm{N}-\mathrm{N} \mathrm{RDF}$, though not providing a shoulder, shows a slightly asymmetric first peak. This feature has been observed in previous simulation where the pair interactions were

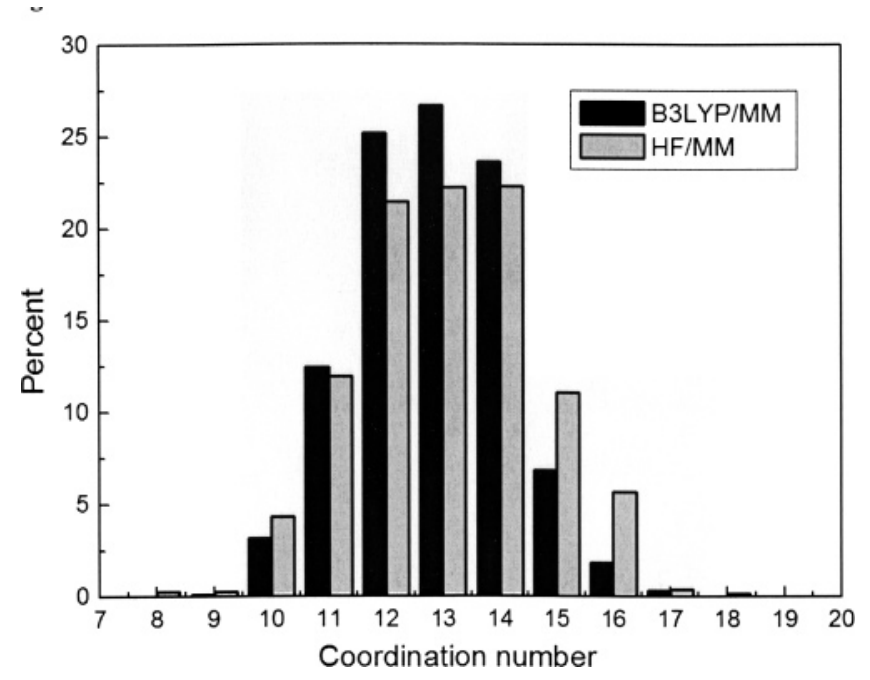

Figure 3. Probability distributions of the coordination numbers, calculated up to first minimum of the $\mathrm{N}-\mathrm{N}$ RDFs.

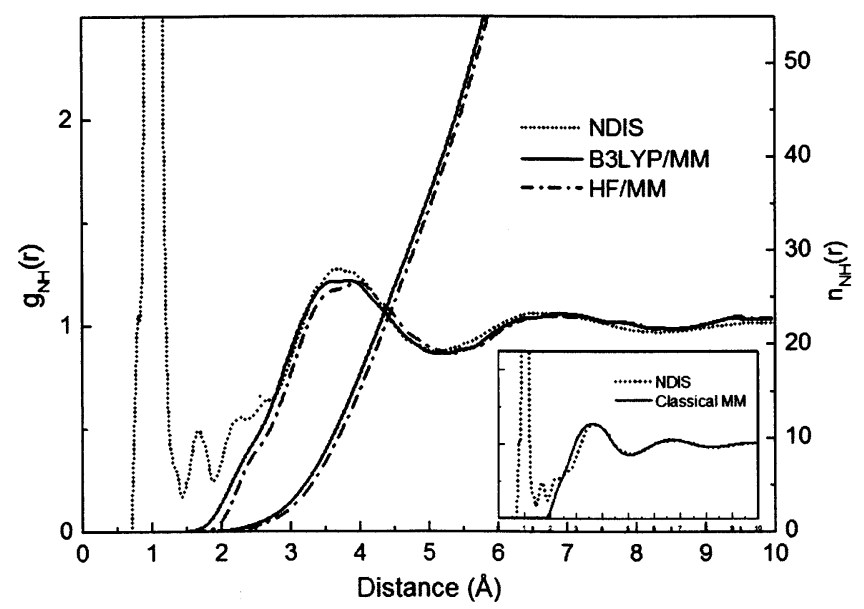

Figure 4. $\mathrm{N}-\mathrm{H}$ radial distribution functions and their corresponding integration numbers, comparing the results obtained from the simulations and the NDIS experiment.

calculated directly using the first-principle ab initio method. ${ }^{15}$ In the X-ray diffraction experiment, ${ }^{4}$ this shoulder has been attributed, on the basis of the solid structure, to the hydrogenbonded molecular pairs. It was proposed in ref 11 that the shoulder is the contribution from the other six ammonia molecules, unless the six closest neighbors located under the first peak at $3.4 \AA$, are lying beyond the first shell to occupy the rest space around the central ammonia.

Figure 3 shows the probability distributions of the number of ammonia molecules surrounding the central reference molecule, calculated up to first minimum in the $\mathrm{N}-\mathrm{N}$ RDFs. In the $\mathrm{HF} / \mathrm{MM}$ simulation, the preferred coordination numbers of 12,13 and 14 are observed in comparable amounts, whereas the B3LYP/MM simulation reveals slight different proportion, with a favored value of 13 (followed by 12 and 14 in decreasing amounts). The broad distribution of the coordination number varying from 10 to 16 coordinated molecules, clearly indicates the disordered close-packing structures of the liquid $\mathrm{NH}_{3}$, in contrast to that of bcc-solid phase.

The characteristics of hydrogen bonds in liquid $\mathrm{NH}_{3}$ can be interpreted through the $\mathrm{N}-\mathrm{H}$ and $\mathrm{H}-\mathrm{H}$ RDFs, shown in Figures 4 and 5 , respectively. In comparison with the $\mathrm{N}-\mathrm{N}$ RDFs, the $\mathrm{N}-\mathrm{H}$ and $\mathrm{H}-\mathrm{H}$ RDFs obtained by the B3LYP/MM simulation are in better agreement with the NDIS data than those of the HF/MM simulation. According to the NDIS data, a distinct 


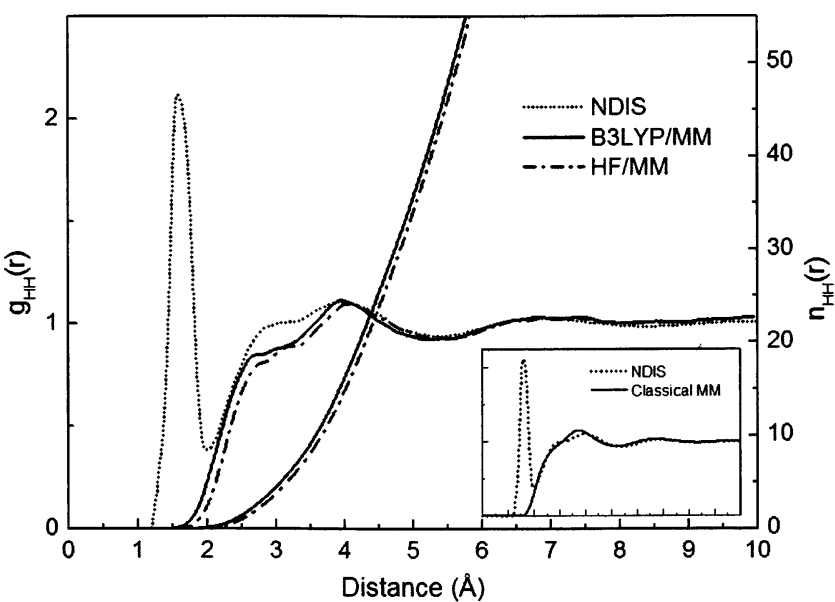

Figure 5. $\mathrm{H}-\mathrm{H}$ radial distribution functions and their corresponding integration numbers, comparing the results obtained from the simulations and the NDIS experiment.

feature of $\mathrm{N}-\mathrm{H}$ RDF at $\sim 2.25 \AA$ was interpreted as hydrogenbond formation in the liquid ammonia. In both HF/MM and B3LYP/MM simulations, this feature is less evident; i.e., only slightly distinct peaks are recognizable at $\mathrm{N} \cdot \cdots \mathrm{H}$ distances between 2.4 and $2.7 \AA$. Therefore, it becomes more ambiguous to evaluate the number of hydrogen bonds in the liquid. Nevertheless, integrations up to a $\mathrm{N} \cdots \mathrm{H}$ distance of $2.7 \AA$ (hydrogen-bond acceptor) in the N-H RDFs yield $1.3(\mathrm{HF} /$ $\mathrm{MM}$ ) and 1.1 (B3LYP/MM) hydrogen atoms being bonded to nitrogen atom of the central reference molecule. Likewise, integrations up to a $\mathrm{H} \cdots \mathrm{N}$ distance of $2.7 \AA$ (hydrogen-bond donor) in the $\mathrm{H}-\mathrm{N}$ RDFs (data not shown) give 1.1 (HF/MM) and 0.9 (B3LYP/MM) nitrogen atoms being bonded to hydrogen atoms of the central ammonia. These data suggest that each ammonia molecule in the liquid has on the average close to one donor and one acceptor hydrogen bonds. With regards to the rather large nearest surrounding molecules $(\sim 13-14)$, the observed numbers of hydrogen bonds in the liquid phase are much smaller, especially when compared to those of liquid water.

To provide a more detailed description of the hydrogen bonds among ammonia molecules, the distributions of $\mathrm{N}-\mathrm{H} \cdots \mathrm{N}$ (donor) and $\mathrm{N} \cdot \cdot \mathrm{H}-\mathrm{N}$ (acceptor) angles, calculated up to the $\mathrm{H} \cdots \mathrm{N}$ and $\mathrm{N} \cdots \mathrm{H}$ distances of 2.7 and of $5.0 \AA$, respectively, are displayed in Figure 6. At the short $\mathrm{H} \cdots \mathrm{N}$ and $\mathrm{N} \cdots \mathrm{H}$ distances of $2.7 \AA$, both the HF/MM and the B3LYP/MM simulations show bent $\mathrm{N}-\mathrm{H} \cdots \mathrm{N}$ and $\mathrm{N} \cdot \cdots \mathrm{H}-\mathrm{N}$ angles, indicating that only weak hydrogen bonds could be exist in the liquid $\mathrm{NH}_{3}$. These findings are different from those of the previous CP-MD studies, ${ }^{17}$ which showed that the linear hydrogen-bond geometry should be preferred in the liquid phase. The preference for linear hydrogen bond observed in the CP-MD studies can be due to the use of simple density functionals, which often yield overestimation of the correlation energy. ${ }^{34}$ Looking at the longer $\mathrm{H} \cdots \mathrm{N}$ and $\mathrm{N} \cdots \mathrm{H}$ distances $(5.0 \AA)$, which are approximately the first minimum of the $\mathrm{H}-\mathrm{N}$ and $\mathrm{N}-\mathrm{H}$ RDFs, the observed broad distributions indicate that most ammonia molecules in the first coordination shell are not hydrogen-bonded to the central reference molecule. These data suggest that the non-hydrogen bonding interactions such as packing or steric effects can play a more important role in determining the solvation behavior of ammonia in the liquid phase, rather than the contributions of hydrogen-bond interactions. The observed weakness hydrogen bonds are in accordance with the NDIS experiment. ${ }^{7}$

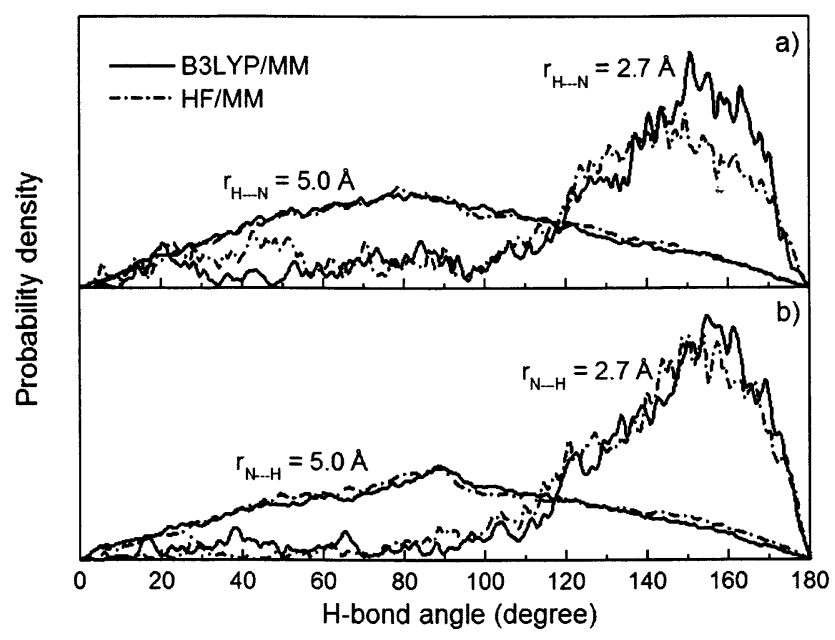

Figure 6. Probability distributions of the (a) $\mathrm{N}-\mathrm{H} \cdots \mathrm{N}$ (donor) and (b) $\mathrm{N} \cdots \mathrm{H}-\mathrm{N}$ (acceptor) angles, calculated within the $\mathrm{H} \cdots \mathrm{N}$ and $\mathrm{N} \cdot$ $\cdot \mathrm{H}$ distances of 2.7 and of $5.0 \AA$.

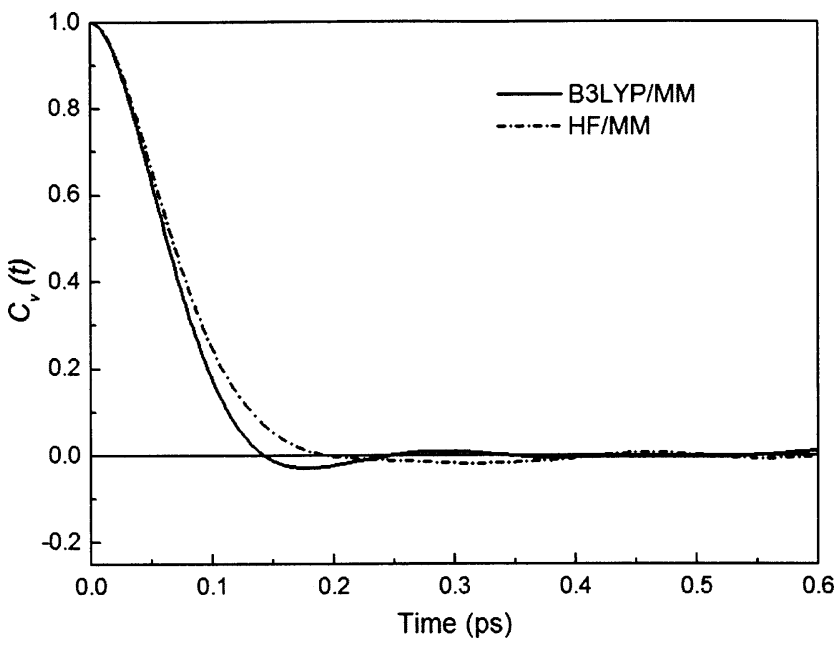

Figure 7. Center-of-mass velocity autocorrelation functions of reference ammonia molecule and all other nearest surrounding molecules involved within the first coordination shell.

3.2. Dynamical Data. Dynamical properties of the liquid $\mathrm{NH}_{3}$ can be obtained from the time correlation functions $\left(C_{\nu}(t)\right)$. Figure 7 shows the center-of-mass velocity autocorrelation functions (VACFs) for the ammonia molecules involved within the QM-treated first coordination shell of reference molecule. In the B3LYP/MM simulation, the VACFs of the ammonia molecules decay to zero faster than those observed in the HF/ MM simulation. This indicates that the inclusion of electron correlation results in higher rigidity of the coordination shell.

The self-diffusion coefficients $(D)$ of ammonia molecules found within the first coordination shell of reference molecule are calculated from the center-of-mass VACF using the GreenKubo relation, ${ }^{41}$

$$
D=\frac{1}{3} \lim _{t \rightarrow \infty} \int_{0}^{t} C_{v}(t) \mathrm{d} t
$$

In this work, the calculated $D$ values are $11.34 \times 10^{-5}$ and $9.96 \times 10^{-5} \mathrm{~cm}^{2} \mathrm{~s}^{-1}$ for the HF/MM and B3LYP/MM simulations, respectively. These values are in good agreement with the experimental value of $8.75 \times 10^{-5} \mathrm{~cm}^{2} \mathrm{~s}^{-1}{ }^{42}$ In the B3LYP/MM simulation, the smaller $D$ value, compared to that of $\mathrm{HF} / \mathrm{MM}$ simulation, is understood to be due to the rigidity of the liquid $\mathrm{NH}_{3}$ structure. The corresponding $\mathrm{MM}$ value 


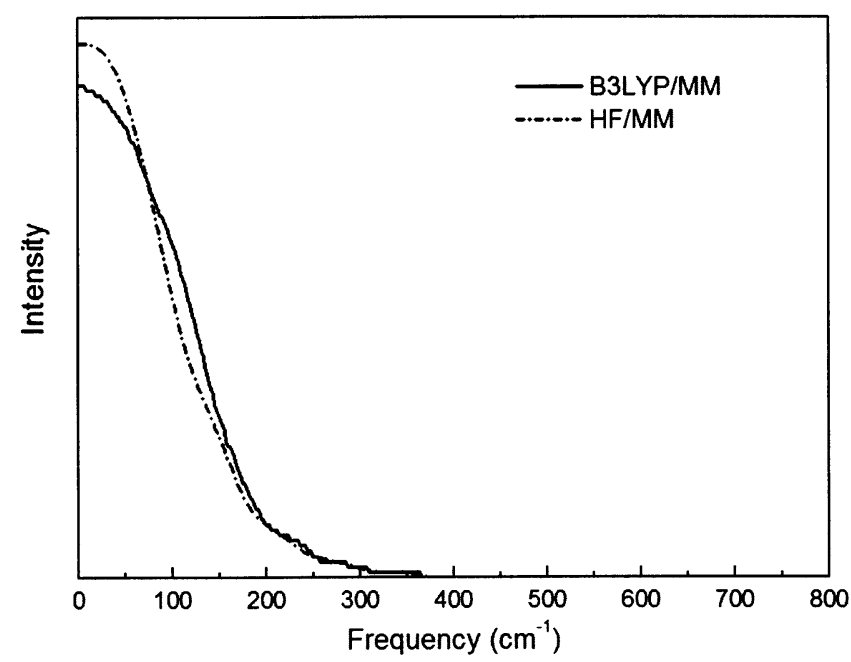

Figure 8. Fourier transforms of the center-of-mass velocity autocorrelation functions of reference ammonia molecule and all other nearest surrounding molecules involved within the first coordination shell.

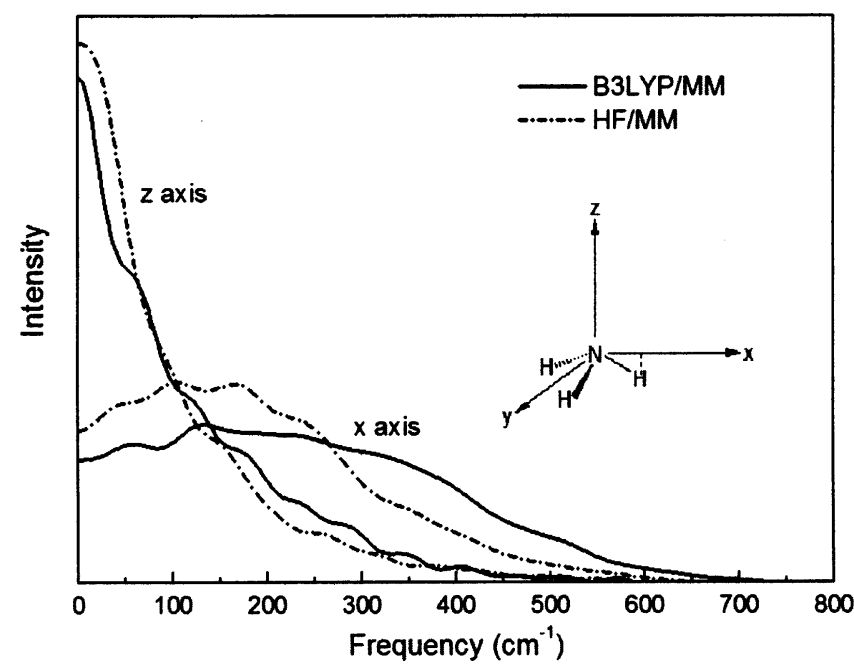

Figure 9. Fourier transforms of the velocity autocorrelation functions of reference ammonia molecule and all other nearest surrounding molecules involved within the first coordination shell.

yielded from the ab initio fitted pair potential simulation ${ }^{11}$ is $9.00 \times 10^{-5} \mathrm{~cm}^{2} \mathrm{~s}^{-1}$, which is rather close to the experimental data. However, it should be noted that this quantity, as well as the structural properties, depends quite strongly on the quality of the potential models employed in the simulations. In this context, the difference between the diffusion coefficients obtained from the QM/MM and the classical (using pair potential) simulations indicates an inadequacy of the pair approximation, which entirely neglects the effects of many-body interactions. The power spectra, which are due to the hindrance of translational motions of liquid $\mathrm{NH}_{3}$, are calculated by Fourier transform of the center-of-mass VACFs, shown in Figure 8. In both simulations, a single band with maximum at zero frequency is observed. This indicates a highly diffusive translational motion of ammonia molecule in the liquid.

With respect to the normal-coordinate analyses, ${ }^{43}$ the power spectra of the librational motions, calculated from the three components of the hydrogen's velocities of all ammonia molecules found within the first coordination shell of reference molecule, are shown in Figure 9. In both the HF/MM and the B3LYP/MM simulations, rotation around the $z$ axis (dipole axis) produces a peak with its maximum at zero frequency. This demonstrates a rather free rotational motion of ammonia
TABLE 1: Mean Residence Times (ps) of Reference Ammonia Molecule and All Other Nearest Surrounding Molecules Involved within the First Coordination Shell

\begin{tabular}{cccccccc}
\hline & & \multicolumn{2}{c}{$t^{*}=0.0 \mathrm{ps}$} & & \multicolumn{2}{c}{$t^{*}=0.5 \mathrm{ps}$} & \\
\cline { 3 - 4 } $\mathrm{CN}$ & $t_{\mathrm{sim}}$ & $N_{\mathrm{ex}}^{0}$ & $\tau_{\mathrm{H}_{2} \mathrm{O}}^{0}$ & & $N_{\mathrm{ex}}^{0.5}$ & $\tau_{\mathrm{H}_{2} \mathrm{O}}^{0.5}$ & method \\
\hline 13.6 & 18.0 & 744 & 0.33 & & 151 & 1.62 & HF/MM \\
13.3 & 14.0 & 483 & 0.38 & & 112 & 1.66 & B3LYP/MM
\end{tabular}

molecules around this axis. For the rotation around the $x$ axis, the B3LYP/MM simulation clearly predicts a greater rigidity of the liquid structure; i.e., the rotation of ammonia molecules around this axis requires slightly more effort than in the case of HF/MM simulation. In comparison to liquid water, ${ }^{28,35}$ it is obvious that the rotational motions of ammonia molecules in the liquid phase are rather fast processes, which contribute to the short-time dynamics of the hydrogen bonds in liquid $\mathrm{NH}_{3}$.

According to the $\mathrm{N}-\mathrm{N}$ RDFs, the nonzero first $\mathrm{N}-\mathrm{N}$ minimum observed in both simulations indicates the lability of ammonia molecules in the liquid phase. In this work, the mean residence times (MRTs), which can be used to measure the exchange processes of ammonia molecules within the QMtreated first coordination shell, are calculated using the direct method $^{44}$ with $t^{*}$ values of 0.0 and $0.5 \mathrm{ps}$. The calculated MRT values are summarized in Table 1 . It should be noted that the MRT data obtained with the $t^{*}=0.0 \mathrm{ps}$ are often used as an estimation of hydrogen-bond lifetimes, whereas the data obtained with $t^{*}=0.5 \mathrm{ps}$ can be considered to be a good measure for exchange processes. ${ }^{44}$ In the B3LYP/MM simulation, slower exchange rates are observed, when compared to the HF/MM results. The tendency of the B3LYP method to slow the dynamics of ammonia molecules in the liquid phase is in good accord with the observed greater rigidity of the solvation structure. Our B3LYP/MM results concerning the dynamics properties of liquid $\mathrm{NH}_{3}$ are quite reliable, unlike that for the case of liquid water, where the B3LYP-level results apparently show too rigid water structure, which results in artificially slow exchange rates. ${ }^{35}$

\section{Conclusion}

In this work, the sophisticated QM/MM simulation technique has been used to obtain detailed structural and dynamical descriptions of liquid $\mathrm{NH}_{3}$ at the molecular level. It is observed that the hydrogen bonds in liquid $\mathrm{NH}_{3}$ are rather weak, and that the structural feature and other related dynamics properties of this liquid are determined by the non-hydrogen-bond interactions, rather than by the hydrogen-bond interactions. With respect to the results obtained from both HF/MM and B3LYP/ MM simulations, all structural and dynamical data are rather equivalent within the methodical limits and are in good agreement with the recent experimental observations. The small differences between the two simulations can be regarded as a consequence of the approximations and the parametrizations of the B3LYP method, which is well-known to describe liquids slightly more rigid than ab initio MO methods.

Acknowledgment. Financial support for this work by the Thailand Research Fund under the TRF Senior Research Scholar Program (Project No. RTA4680008) is gratefully acknowledged. We thank A. K. Soper for providing the experimental neutron diffraction data on liquid ammonia and Professor I Ming Tang for reading the manuscript.

\section{References and Notes}

(1) Schuster, P.; Zundel, E.; Sanfordy, C. The Hydrogen Bond; NorthHolland: Amsterdam, 1976. 
(2) Jeffrey, G. A. An Introduction to Hydrogen Bonding; Oxford University: New York, 1997.

(3) Wiggins, P. M. Microbiol. Rev. 1990, 54, 432.

(4) Narten, A. H. J. Chem. Phys. 1977, 66, 3117.

(5) Chieux, P.; Bertagnolli, H. J. Phys. Chem. 1984, 88, 3726.

(6) Postorino, P.; Ricci, M. A.; Soper, A. K. J. Chem. Phys. 1994, $101,4123$.

(7) Ricci, M. A.; Nardone, M.; Ricci, F. P.; Andreani, C.; Soper, A. K. J. Chem. Phys. 1995, 102, 7650.

(8) Jorgensen, W. L.; Ibrahim, M. J. Am. Chem. Soc. 1979, 102, 3309.

(9) Kincald, R. H.; Scheraga, H. A. J. Phys. Chem. 1982, 86, 833

(10) Impey, R. W.; Klein, M. L. Chem. Phys. Lett. 1984, 104, 579.

(11) Hannongbua, S. V.; Ishida, T.; Spohr, E.; Heinzinger, K. Z Naturforsch., A: Phys. Sci. 1988, 43, 572.

(12) Gao, J.; Xia, X.; George, T. F. J. Phys. Chem. 1993, 97, 9241.

(13) Kiselev, M.; Kerdcharoen, K.; Hannongbua, S.; Heinzinger, K. Chem. Phys. Lett. 2000, 327, 425.

(14) Beu, T. A.; Buck, U. J. Chem. Phys. 2001, 114, 7848.

(15) Hannongbua, S. J. Chem. Phys. 2000, 113, 4707.

(16) Diraison, M.; Martyna, G. J.; Tuckerman, M. E. J. Chem. Phys. 1999, 111, 1096.

(17) Boese, A. D.; Chandra, A.; Martin, J. M. L.; Marx, D. J. Chem. Phys. 2003, 119, 5965.

(18) Car, R.; Parrinello, M. Phys. Rev. Lett. 1985, 55, 2471.

(19) Marx, D.; Hutter, J. Modern Methods and Algorithms of Quantum Chemistry; Grotendorst, J., Ed.; NIC: FZ Jülich, 2000.

(20) Liu, Y.; Tuckerman, M. E. J. Phys. Chem. B 2001, 105, 6598.

(21) Warshel, A.; Levitt, M. J. Mol. Biol. 1976, 103, 227.

(22) Singh, U. C.; Kollman, P. A. J. Comput. Chem. 1986, 7, 718.

(23) Field, M. J.; Bash, P. A.; Karplus, M. J. Comput. Chem. 1990, 11 , 700 .

(24) Gao, J. Rev. Comput. Chem. 1996, 7, 119.

(25) Rode, B. M.; Schwenk, C. F.; Tongraar, A. J. Mol. Liq. 2004, 110 ,
(26) Schwenk, C. F.; Loeffler, H. H.; Rode, B. M. J. Chem. Phys. 2001 $115,10808$.

(27) Kerdcharoen, T.; Liedl, K. R.; Rode, B. M. Chem. Phys. 1996, 211,313 .

(28) Tongraar, A.; Liedl, K. R.; Rode, B. M. J. Phys. Chem. A 1997 101,6299

(29) Tongraar, A.; Liedl, K. R.; Rode, B. M. J. Phys. Chem. A 1998, 102,10340 .

(30) Tongraar, A.; Sagarik, K.; Rode, B. M. J. Phys. Chem. B 2001, $105,10559$.

(31) Tongraar, A.; Sagarik, K.; Rode, B. M. Phys. Chem. Chem. Phys. 2002, 4, 628 .

(32) Tongraar, A.; Rode, B. M. Phys. Chem. Chem. Phys. 2003, 5, 357.

(33) Tongraar, A.; Rode, B. M. Chem. Phys. Lett. 2005, 403, 314.

(34) Intharathep, P.; Tongraar, A.; Sagarik, K. J. Comput. Chem. 2005, $26,1329$.

(35) Xenides, D.; Randolf, B. R.; Rode, B. M. J. Chem. Phys. 2005, 122,174506

(36) Hannongbua, S. Aust. J. Chem. 1991, 44, 447.

(37) Dunning, T. H., Jr.; Hay, P. J. Modern Theoretical Chemistry; Schaefer, H. F., Ed.; Plenum III: New York, 1976.

(38) Brooks, B. R.; Bruccoleri, R. E.; Olafson, B. D.; States, D. J.; Swaminathan, S.; Karplus, M. J. Comput. Chem. 1983, 4, 187.

(39) Berendsen, H. J. C.; Postma, J. P. M.; van Gunsteren, W. F.; DiNola, A.; Haak, J. R. J. Phys. Chem. 1984, 81, 3684.

(40) Adams, D. J.; Adams, E. H.; Hills, G. J. Mol. Phys. 1979, 38, 387.

(41) McQuarrie, D. A. Statistical Mechanics; Harper \& Row: New York, 1976.

(42) O'Reilly, D. E.; Peterson, E. M.; Scheie, C. E. J. Chem. Phys. 1973, 58,4072 .

(43) Bopp, P. Chem. Phys. 1986, 196, 205.

(44) Hofer, T. S.; Tran, H. T.; Schwenk, C. F.; Rode, B. M. J. Comput. Chem. 2004, 25, 211. 